\title{
Psycosmogenic Patterns: A Premise for Environmental Harmony
}

Ardita Byci Jakupi ${ }^{1}$

\begin{abstract}
The interest in holism is leading to new ways for development of multi-layered skills working towards a holistic integrated approach and developing new ways of multidimensional and multilayered conceptualization of space. When the making of the place is requested, urban designers must be able to sense and interpret the visible and invisible dimension of place, in order to come to an understanding which leads to the holistic approach. Especially knowing how to recognize the invisible dimension of the site and knowing how to visualize it is one of the main challenges. This doesn't depend only on methods or tools, but also on creativity and imagination that has a relation with experience, culture or knowledge of the architect. The following is important in most of the cases: intuition, sensitivity for the unseen, balance between the imagination, truth and the world around us. The aim of this research is to show that architects must perceive the site as a living being, as a unity of aspect of consciousness and essence, aspect of energy and vitality and aspect of physical form. Through this approach, by understanding and using of invisible patterns, we can generate sustainable concepts that lead towards environmental harmony.
\end{abstract}

Keywords: Invisible patterns, perceiving, sensing, concept, environmental harmony.

\section{Introduction}

In these times, that we are living, there is a kind of isolation between man and nature. The results of this materialistic point of view in general can be seen with imbalance of environment. Cities are growing without any connection with the spirit of the place, a lot of buildings and objects are placed without consideration for aesthetics or quality. Earth and land as spiritual beings remain ignored.

On the other side architects are trying to use holistic integrated approach to develop multidimensional conceptualization of space. If holistic approach means inclusion of all dimensions of the place: morphological, functional, visual, social, perceptual and temporal, then there is a strong platform to do, to plan, and to design something where everything is in the right place, that means to create harmonious environments. If holism means this, why are we constantly facing these environments that give the impression of disharmony, discordance and imbalance? Is it about miss sensing of the site, is it about the lack of the deep analysis of the site, or is it just a cultural way of seeing?

Our world needs urgently a spiritual point of view, which reconnects man and nature, which relates human culture with the power of the nature, which understands cities and landscapes as an organic unit and makes visible the aspect of nature. 


\section{Invisible Patterns - Psycosmogenic Patterns}

Different environments, different landscapes present different patterns which we see and experience. One of the challenges for architects and urban designers during the design phase is how to perceive environment and landscape as composition of several visible and invisible patterns and how they could be used accordingly.

Patterns are evident at very wide and large scales from the molecular structure of the DNA at microscopic level, to spirals in the universe. Patterns are of different ages and can be classified in four categories.

Primary-natural patterns are found in the existing landscape, resulting from flows of energy from geology, from the nature of materials, from the processes of growth and decay. Secondary or human patterns are found in urban and rural landscape. They can be results of human behavior who adapt the places to satisfy the needs for food, shelter, transport, comfort and security.

Tertiary -aesthetic patterns result from artist's imagination or the aesthetic appreciation of nature. They may derive from geometry, mathematics, decoration, mythology, representation, symbolism, allegory, metaphor, abstraction, philosophy, poetry, music and narrative.

Quaternary or archetypal patterns are tried and tested combinations of the other patterns. They are prototypes that have proved successful. Like plant associations, house types, farm type and settlement types. Their place in outdoor design, which is a sitespecific art, is as components (Turner, 1996).

The importance of senses in architecture has been mentioned in several researches which express their significance for our experience and understanding the world. The role of the body as a locus of perceptions, thoughts and consciousness and the significance of the senses in articulation, storing and processing responses and thoughts have been strongly confirmed. In the „Questions of Perceptions“ (Holl et al, 2007), Holl speaks about the integration of sensory perception as a function of build form, in other words implementing sensory design in order to establish experiential, architectural space. This establishes the necessity for place making through sensory observance. According to Turner's patterns of categorization and Holl's phenomenological concept strategies, there can be seen that almost all patterns are included in certain groups and we could be able to easily categorize a particular pattern. But what about the patterns which can not be explained in material terms, that has to do with the nature of existence, or the nature of consciousness. Patterns related to the essence, subtle dimensions and beings.

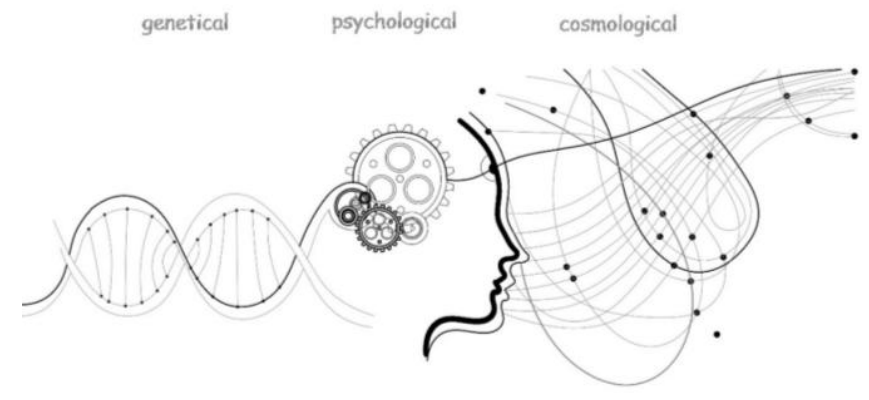

Figure 1: Invisible patterns 
I have called this group of patterns Psycosmogenic patterns (psychological, cosmic and genetic) (Figure 1). Psychological - which is related with the work of mind; Cosmic which is a result of individual and cosmic resonance, an expression of the totality of natural laws; Genetic - that we are genetically programmed to feel a certain need (for example, we like nature because we are genetically programmed to live in a natural habitat).

Psychological patterns are formulated as additional patterns that can be seen, felt or admired in the natural or human environment. It might find similarities with other patterns but with this category of invisible patterns there is always a difference.

Psychological patterns are those patterns which are based in: perception, motivation, emotion, learning, intelligence and personality and innate patterns

Genetic patterns are related with physiological, biological, psychological, biochemical, physical and social needs.

Cosmological patterns are composed from: consciousness, spirituality, transcendence, psycho-physical being, quantum mechanics etc.

Psycosmogenic patterns present a whole constellation of human needs, desires, fantasies, doubts, feelings and perceptions (Figure 2). They can be very abstract, coldly logical, poetic, emotional or passionate, that is why it is a big challenge to interpret or visualize it. Advantages of recognition of these patterns start with a simple practical issue that has to do with the understanding of subtle patterns that form so much our daily life.

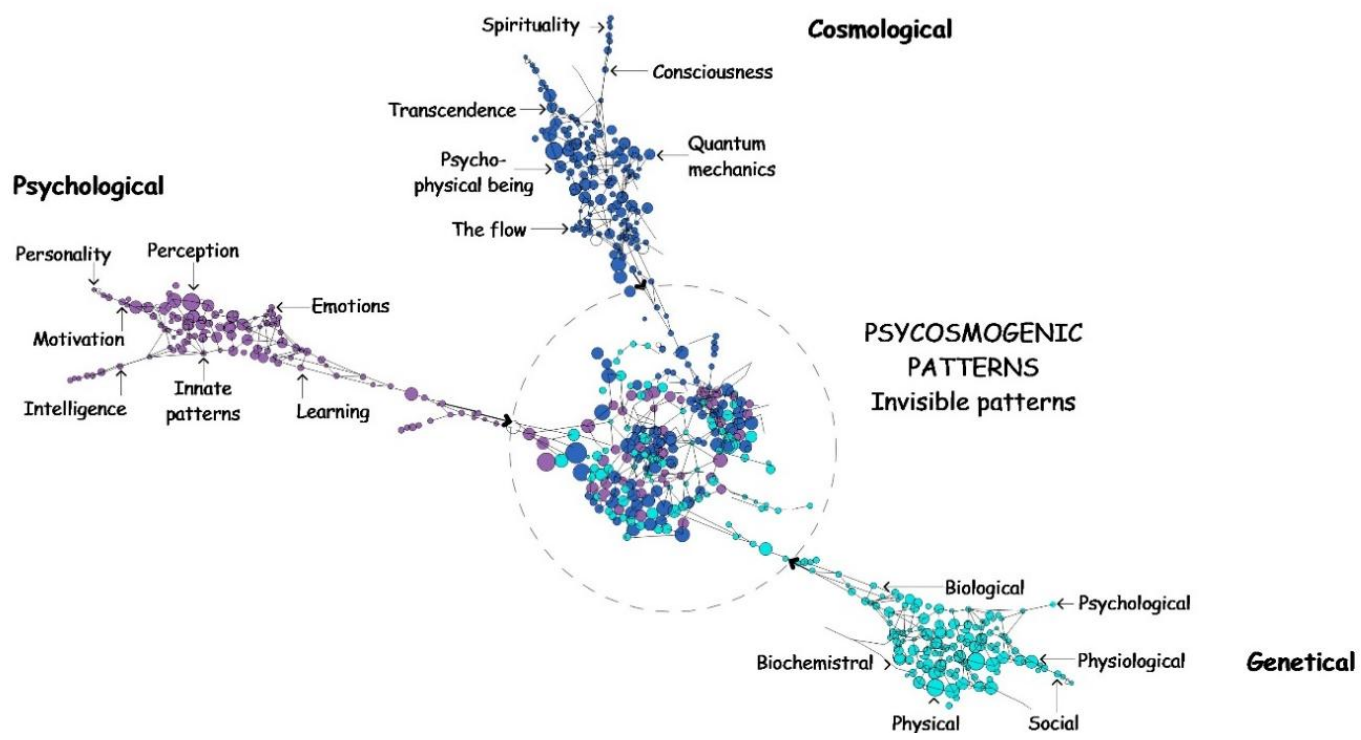

Figure 2: The building of psycosmogenic patterns

Perception of the site as e living being may be an approach which can help in bringing this dimension much closer. The site should be seen as a unity composed from three aspects: aspect of consciousness and essence (anima or soul), aspect of energy and vitality (spirit or life), and aspect of physical form (corpus or body).

Analogies and philosophical approaches to life for some societies, when sensitivity for 
the unseen and spirituality is part of their everyday life, can help in bringing a lent of viewing/ 'seeing' and teaching us to interpret the complex patterns of particular sitepsycosmogenic patterns.

\section{A Lent of Viewing}

Sometimes to understand things we have to find indirect ways or creative approaches that maybe are not immediately obvious. Using intuition, imagination through unconscious and subconscious processes best fits if we think in a lateral way.

As humans we have created ways to explain the mysteries of the world. We have codified our existence by bringing it to a human scale so that we can understand it more easily. We have invented mathematics as a concept - a human production, an artificial construction only to be able to understand the universal truth.

\subsection{Perception, Emotion and Experience}

"Touch has a memory", John Keats

Perception is organization, identification and interpretation of sensory information in order to represent and understand the environment. Perception is composed from knowledge, learning, memory, expectations and attention of the observer that is why the interpretation is very personal and it depends from the sense of perception.

In the field of urbanism perception of the space is one of the main or essential dimensions. Since the 60-s it has been developed as an interdisciplinary field for environment perception that mostly includes symbolism and the meaning of the place and especially the sense of place.

The four most valuable senses in interpreting and sensing the environment are vision, hearing, smell and touch. (Carmona et al, 2004)

Visual perception is highly complex, relying in distance, color, shape, textural and contrast gradients etc. While visual space involves what lies before us, 'acoustic' space is all of the surrounding, and has no obvious boundaries and emphasizes space itself.

The sense of smell, although not very rich emotionally than other senses, it plays a role in perception of the space. Touch, in urban context can be experienced through our feet, or when we sit down.

Perception sometimes referred as 'cognition' concerns more than just seeing or sensing the urban environment. According to Ittelson "it refers to more complex processing or understanding of stimuli" (Carmona et al, 2004): cognitivity (which involves organizing and keeping information); affectivity (feelings which influence perception of the environment); interpretation (meaning derived from the environment) and evaluability (values and preferences).

On other side, Lynch through cognitive geography describes how aspects of environment can leave a strong image in the mind of the observer. Paths, edges, districts, nodes and landmarks are five key physical elements that define an image of the site. But there is a lack of using this sample because the perception of environment depends on the observer, legibility, meaning and symbolism.

Emotions - Experience plays a huge role during the survey or analysis of certain sites. It may have different implications that depend on the experience coherence which is why 
the results of a survey may be different from different people. Experience can be: physical, mental, emotional, spiritual, social, subjective etc.

Physical experience has to do with observation, while social experience is more shared experience that has to do with tradition, values, social role, symbols and language.

In context of the sense of the place, emotional, mental and spiritual experience has more influence in this perception. Mental experience involves intellect and conciseness as a combination of thought, perception, memory, emotion, wishes and imagination whether emotion experience has to do with that how people can rationalize. This involves the emotional states, for example: happiness, sadness, love, pregnancy etc.

Among these type of experiences, spiritual experience is very interesting since it 'coats' the experience with another hue, sensing another dimension- the dimension of universal harmony that has to do with the flows of the nature or the energy of the place. As a different state of consciousness, it involves all senses in different level of experience. That is why people or architects who have a strong spiritual experience are able to see more invisible patterns than other people.

\subsection{Philosophical Systems and Analogies}

Maharishi Vedic Architecture is known as the oldest and most complete planning system. Architecture and planning are based on solar, lunar and planetary influences on earth with reference on north and south poles and the equator- connecting individual life with Cosmic life, individual intelligence with Cosmic intelligence, and in this way creating ideal living conditions.

According to Vedic's principles, a sustainable city is a city where everyone is happy, healthy, wealthy and wise. Only a city that is free from pollution, noise and stress and which is designed as a holistic structure in harmony with natural law, where individual life is flowing with evolutionary stream of energy and intelligence is really sustainable.

The main goal is to design in harmony with natural law, in the way to create living environments that are ideal for the inhabitants and the planet.

Ancient cultures were very closely related to nature, while some cultures in a passive and a silent way were dealing with natural phenomena. Other high cultures such as Chinese geomancy were trying to reshape their cultural life and architectonic expression in harmonious ways.

Geomancy means interpreting the visible and invisible dimensions of places and landscapes, so that a holistic understanding of their true essence can emerge in our consciousness. It is a kind of a mixture of imaginary, feeling qualities, symbolic images, sounds, movements. It is a synergic combination of ecology, art and spiritual approach to life.

The main goal of geomancy is to improve relationships between people and their environment based on the concept of protection, education and sustainable development, to increase people's ability to efficiently use Earth, its resources and landscapes.

Since the knowledge of modern geomancy can be used in the area of nature protection, landscape design, architecture and planning, then we can use it as the art of placing or arranging buildings or other sites propitiously and in this way to contribute in creating harmonious environment. 
Empathy- empathy as a process that builds a connection between people is a state of perception without prejudice. Empathy means to 'read' the inner state of the person and to interpret it in a way that could help the other person by offering full support and developing common trust. Empathy in translation to German word "einfühlung" means "to feel as a one" which means to "walk in another's shoes" in order to understand properly his perspective.

In the same way we should act with the site or the building. We should "read" their inner state, as an intuitive act by giving to it all experience and attention. Through an empathic approach the 'depth' of space and building can be felt. And when we stand in front of the site, then we will think longer about the ideas and embody them with it.

Transcendental meditation - Sometimes it needs higher level of understanding and seeing the site. In transcendental meditation the theory of sound (mantra) are the vibrations that harmonize and suit the man with nature and the way of life. The collaboration between individual and universal mind according to transcendental meditation concepts leads to universal harmony.

In this way through TM tendencies when spirituality is combined with physics, absolute with relative, meditation with physical activities, then there is a perception that leads towards a complete harmony. Through objectives of TM as a science of being and art of life, we can experience and understand the subtle meaning of the place.

Philosophical approaches and analogies as explanatory modes to interpret beyond descriptive or expressive way can help to facilitate visualization of psycosmogenic patterns by giving to it a physical - imaginative creation.

\section{An Approach of Sensing}

Every site requires different methods and interpretations of their patterns. Each method has its strengths and limitations but mostly it depends on creativity and imagination of the architect. Difficult to define, but in most of the cases these are very important: intuition, sensitivity for the unseen, balance between the imagination, truth and the world around us. We can develop different ways and languages to describe and analyze them but all this sense of perception has to do with personal interpretation.

One of the ways or an approach for sensing, perceiving the site is an approach which is related with spirituality and physical form- a combination of visual vocabulary and organic design process (Figure 2).

The simplest way to see the complexity of site is through Euclidian shapes. Since patterns appear in different forms and the way we perceive them we should have in consider ways of analyzing them. According to Bell "we can treat them as one of the four basic elements- point, line, plane, volume and combination of them" (Bell, 2004) We should understand the attribute of each basic element before we know how they interact and effect different variables and their spatial organization that produced that particular pattern. So it is a combination of these components; basic elements, its variables and their mode of organization which describes the patterns to be found in the existing place. 


\section{Spiritual $\longrightarrow$ Organic design proccess}

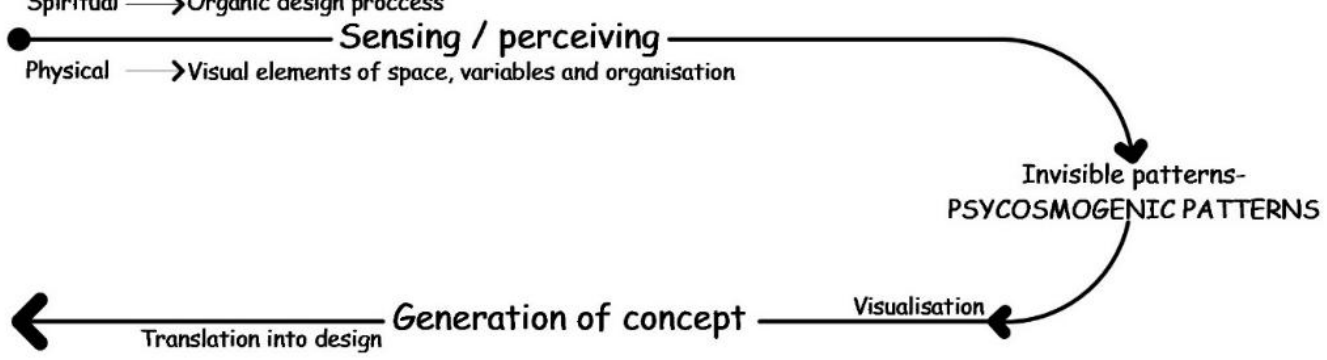

the process of usage

Figure 3: The process of identification and usage of psycomogenic patterns

The main components of the visual vocabulary according to Bell (Figure 4) are:

1. BASIC ELEMENTS: Point, line, plane, solid volume, open volume

2. VARIABLES: Number, position, direction, orientation, size, shape interval, density, color, time, light, visual force, visual inertia

3. ORGANISATION:

- Objectives: diversity, unity, genius loci

- Spatial cues: nearness, enclosure, interlock, continuity, similarity, figure and ground

- Structural elements: balance, tension, rhythm, proportion, scale

- Ordering: axis, symmetry, hierarchy, datum, transformation.

We should use the elements or the combination of them, as separated ones or as part of other element. For analyzing purposes we should understand the attribute of each basic element before we know how they interact and effect different variables and their spatial organization that has produced that particular pattern.

To summarize, basic elements are the basic mass space elements of visual expression. Basic elements can be seen in relation to light, color, time and movement. We see them in many different ways. There is however, a limited but fundamental number of ways of varying them.

On other side, as Garret sad about the spatial organization, "inherent in the structure of the mind is the tendency to organize what we see. It is so strong that the slightest indications of possible connections are enough to cause the perception of a connected path or complete form" (Bell, 2004). 

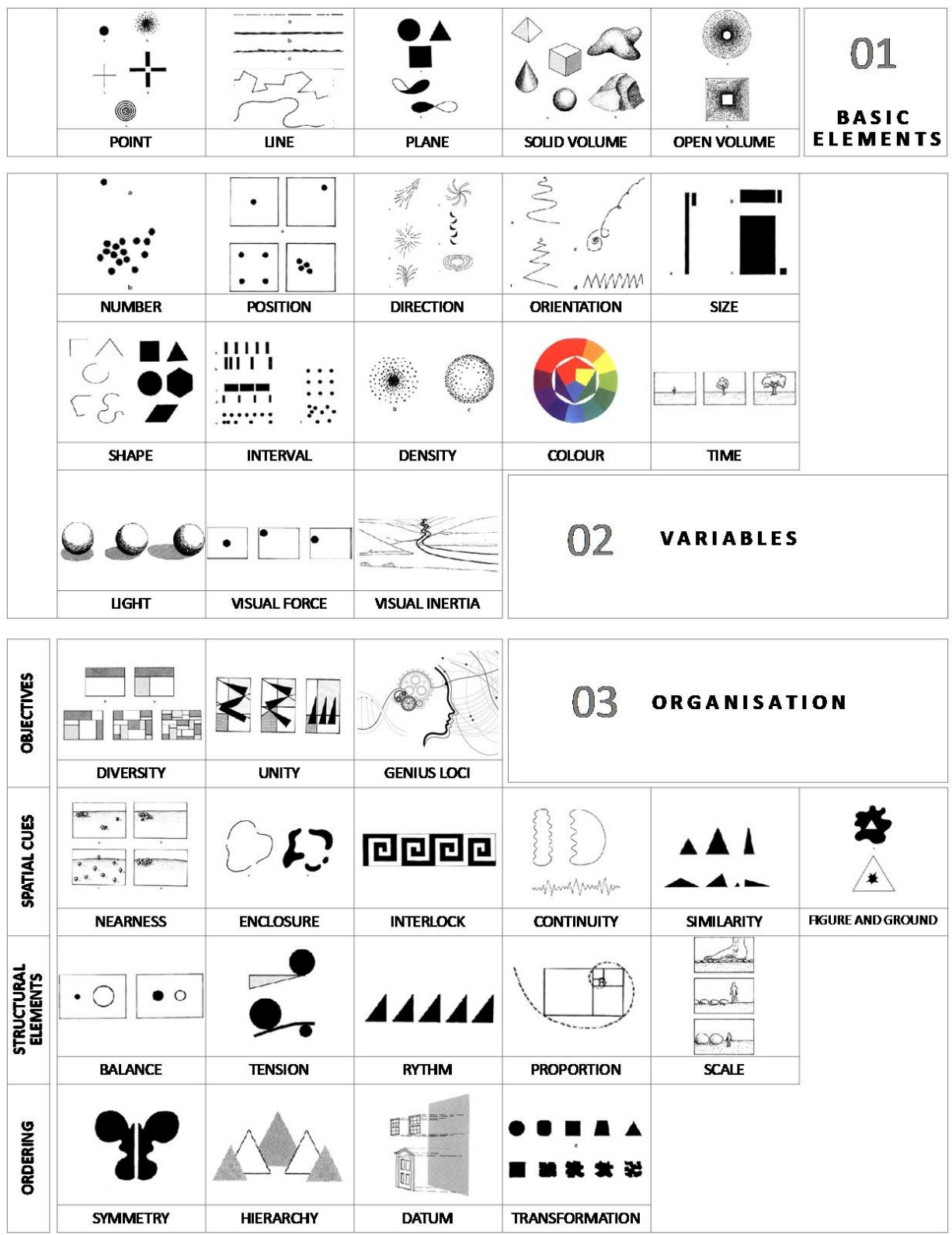

Figure 4: Visual vocabulary of space (based on Bell's "Elements of visual design in landscape" - simplified and intervened from the author)

\subsection{Organic Development of Place}

A pattern that brings a feeling of harmony can be a result from the organization of basic elements in their endless variations, by balancing, for example, the unity with the diversity. But it is necessary to take into account concepts such as genius loci.

Ancient cultures have been aware about this concept since they were very closely related 
to nature. A lot of cultures have reshaped their cultural life and architectonic expression in harmonious ways. Their spiritual approach to life presents a synergic combination of ecology, art and natural laws. That is why for a unified design with environmental harmony as a result, a complex scientific approach with intuitive, holistic and multidimensionality needs to be done.

One of the approaches is the organic development of site. In the book „Spirit and Place“, Day (2003) shows us how to work with different levels of situations: the emotional, continuum-based, underlying essence as well as the practical and rational, since environments affect us both in social and personal health. All aspects of our environment work on us through all of our senses and on all levels of our being, and at three levels of social scale: personal, cultural and universal.

Organic design process is a design process based on four layers of the site: facts, the solid objects, the "bedrock" of the place; that which is constantly changing, flowing and growing, that which lends character to the place, gives its uniquess, 'atmosphere' and appeal, that which is the essence or inner reality of the place (Day, 2003).

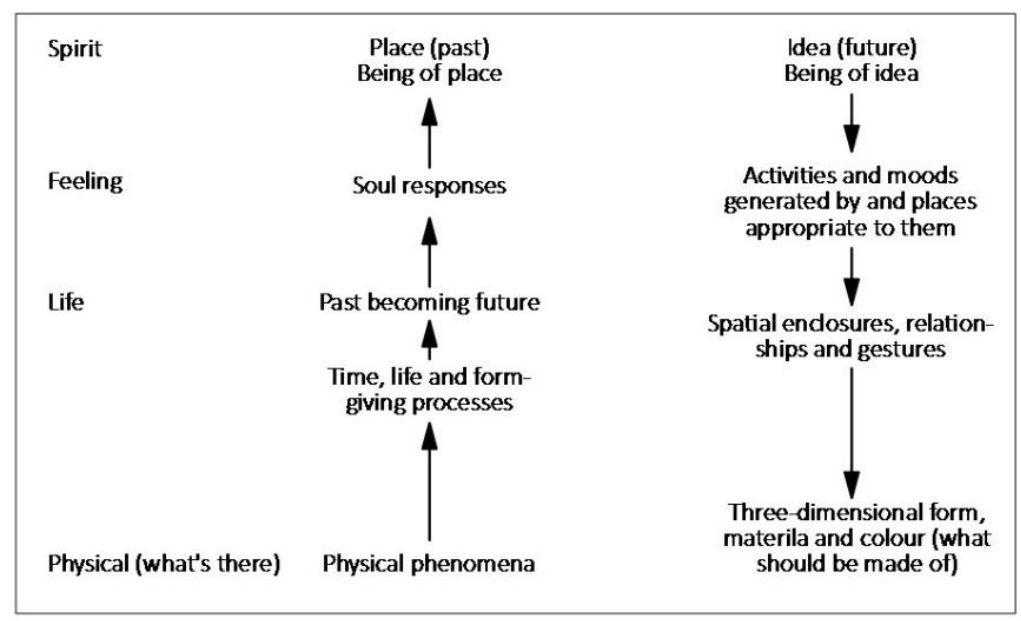

Figure 5: Organic development of place (Day, 2003)

Organic design process is one of the approaches that closes the gap between the thought and the nature and by teaching us how to walk around the place, silently listening to it and opening ourselves, refrains our interferences, thoughts and ideas (Figure 5). It helps us to "record" the place through careful observation and next to describe the mood of sub-places and feelings invoked in us.

Combination of visual vocabulary of the space and organic design process can help in 'decoding' the invisible patterns of the site and use them accordingly in generating a sustainable design concept which leads to environmental harmony.

\section{Conclusion}

The isolation between man and nature results with materialistic point of view, ignoring the spirit of the place. Our world needs a spiritual point of view, which 
reconnects man and nature, which relates human culture with the power of the nature, which understands cities and landscapes as an organic unit and makes the aspect of nature visible.

Architects must perceive the site as an organic unit, as a composition of visible and invisible patterns. Seeing the site not only as a physical or social phenomenon but also as a spiritual one and using a complex holistic approach can help to facilitate the visualization of psycosmogenic patterns and using it as a premise for generation of concepts that leads toward environmental harmony.

\section{References}

Turner, T (1996). City as Landscape- A post-postmodern view of design and planning. 1st ed. E\&FN Spon, Oxford, 34.

Day, Ch (2003). Spirit \& Place-Healing Our Environment. Architectural Press, Oxford, 165-167.

Holl, S., Pallasma, H., Perez-Gomez, A (2007) Questions of Perception: Phenomenology of Architecture. William Stout Publisher, San Francisco.

Bell, S (2004).Elements of Visual Design in Landscape 2nd ed. Spon Press London, 17-107.

Carmona, M., Heath T., Oc T., Tiesdell, S., (2003) .Public Places-Urban Spaces. UK: Architectural Press, 87. 